\title{
Probing microscopic models for system-bath interactions via parametric driving
}

\author{
Anastasia S. D. Dietrich, ${ }^{1, *}$ Martin Kiffner, ${ }^{1,2}$ and Dieter Jaksch ${ }^{1,2}$ \\ ${ }^{1}$ Clarendon Laboratory, University of Oxford, Parks Road, Oxford OX1 3PU, United Kingdom \\ ${ }^{2}$ Centre for Quantum Technologies, National University of Singapore, 3 Science Drive 2, Singapore 117543
}

(Received 21 March 2018; published 18 July 2018)

\begin{abstract}
We show that strong parametric driving of a quantum harmonic oscillator coupled to a thermal bath allows one to distinguish between different microscopic models for the oscillator-bath coupling. We consider a bath with an Ohmic spectral density and a model where the system-bath interaction can be tuned continuously between position and momentum coupling via the coupling angle $\alpha$. We derive a master equation for the reduced density operator of the oscillator in Born-Markov approximation and investigate its quasisteady state as a function of the driving parameters, the temperature of the bath and the coupling angle $\alpha$. We find that the driving introduces a strong dependence of the time-averaged variance of position and momentum on these parameters. In particular, we identify parameter regimes that maximize the $\alpha$ dependence and provide an intuitive explanation of our results.
\end{abstract}

DOI: 10.1103/PhysRevA.98.012122

\section{INTRODUCTION}

Accurate models for the interaction between a quantum system and its environment have been vital for the success of quantum optics and cold atom systems. For example, they have paved the way for revolutionizing methods such as laser cooling [1], and are essential for developing applications in quantum technologies where decoherence effects need to be as small as possible. For some systems our understanding of the system-bath coupling is so accurate that it can be used to control and engineer these interactions. In this way, the role of dissipation and decoherence can be transformed from a detrimental effect into a wanted feature as shown, e.g., in [2-8].

This is in stark contrast to condensed matter systems where the exact microscopic model underlying the system-bath coupling is often unknown [9], and thus one has to resort to phenomenological models. Driving these systems with intense terahertz radiation opens up unprecedented possibilities to manipulate their quantum dynamics [10-23]. Examples include the melting of charge density waves [10-12], the generation of synthetic magnetic fields [13], the control of heterointerfaces [14-16], the possibility to drive metalinsulator transitions $[17,18]$, the parametric cooling of bilayer cuprate superconductors [19], the control of transport modes in cuprate superconductors [20], or even the controlled creation of transient superconductivity [21-23]. In order to optimize the coherent control of these systems even further, it would be highly desirable to improve our understanding of their system-bath interactions.

A direct manifestation of system-bath interactions are decay and decoherence rates that can be directly observed in an experiment. However, these quantities reveal little about the microscopic details of the system-bath interaction. The development of techniques that go beyond measuring decay and decoherence rates is a highly desirable yet difficult task, and only few schemes are known to date. For example, some

\footnotetext{
*anastasia.dietrich@physics.ox.ac.uk
}

information about the local spectral density of a condensed matter heat bath can be obtained by observing the nonMarkovian behavior of an optomechanical resonator coupled to it [24]. A scheme for determining the spectral density based on dynamical decoupling has been developed in [25], and Ref. [26] suggests measuring the noise spectrum using a multiple-pulse sequence.

Recently, it has been shown that strongly driven systems can enter the intriguing regime of environment-governed dynamics [27] (EGD) where the system dynamics is predominantly determined by the details of the system-bath interaction, e.g., the form of the coupling operator. This strongly driven regime can be described within the framework of the Floquet-Markov master equation approach [28-33], which combines ideas from open quantum systems with Floquet theory [34-36]. A fascinating question is thus whether the environment-dependent dynamics in the regime of EGD can be employed to infer microscopic details about the system-bath interaction.

Here we consider a paradigmatic example for system-bath interactions comprising a parametrically driven oscillator that is weakly coupled to a thermal bath; see Fig. 1. The system-bath interaction $H_{I}^{\alpha}$ in our model can be tuned continuously from position to momentum coupling via the parameter $\alpha$. If the driving is switched off, all observables are approximately independent of $\alpha$ since we consider the regime of weak systembath interactions. Note that in this regime, the dynamics of the corresponding classical system is also approximately independent of $\alpha$. In particular, this observation rules out the possibility of determining $\alpha$ by preparing the system in a coherent state and observing its subsequent time evolution.

Here we show that strong parametric driving of the oscillator can lead to a strong $\alpha$ dependence of physical observables via EGD. This dependence allows one to distinguish between microscopic models for the system-bath interaction. More specifically, we derive a master equation for the reduced density operator of the oscillator in Born-Markov approximation and consider the time-averaged variance of position and momentum. We find that these observables exhibit a strong dependence on $\alpha$ due to the parametric driving and identify 


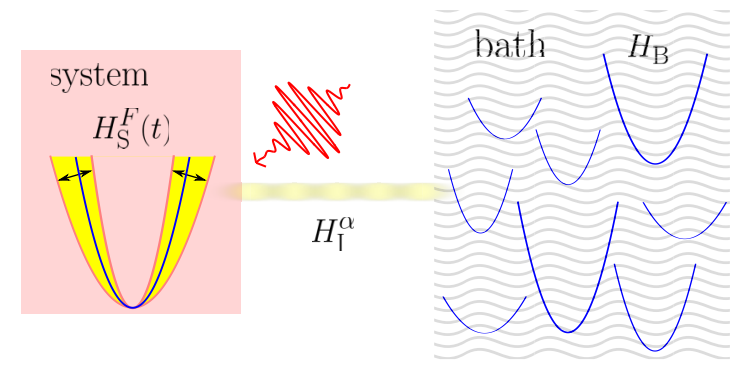

FIG. 1. The system of interest comprises a parametrically driven harmonic oscillator and a bath of harmonic oscillators with Hamiltonians $H_{\mathrm{S}}^{F}(t)$ and $H_{\mathrm{B}}$, respectively. The system-bath interaction is described by the interaction Hamiltonian $H_{I}^{\alpha}$, where the parameter $\alpha$ allows one to continuously adjust the interaction from position ( $\alpha=0$ ) to momentum coupling $(\alpha=\pi / 2)$. The parametric driving affects both the system and dissipation mechanism as indicated by the red arrow.

parameter regimes that maximize their $\alpha$ dependence. We find that our results can be understood in terms of an effective model with an $\alpha$-dependent spectral density that is probed at different Floquet quasifrequencies. This also shows that strong driving allows investigating other aspects of system-bath interactions that go beyond measuring the value of $\alpha$.

The paper is organized as follows. In Sec. II we present our model and discuss the steps and approximations leading to a master equation applicable to a strongly driven system. The presentation of our results in Sec. III begins with a description of the observables that we use to characterize the quasisteady state of the oscillator in Sec. III A. Our numerical results for the time-averaged mean values of the system quadratures as a function of the coupling angle, bath temperature, and driving parameters are presented in Sec. III B. In Sec. IV, we introduce a unitary transformation of the total Hamiltonian in order to explain the $\alpha$ dependence of our results. Finally, we conclude with a summary and an outlook for further work in Sec. V.

\section{MODEL}

Here we describe our theoretical model for a driven harmonic oscillator coupled to a thermal bath. The isolated system is described in Sec. II A, and Sec. II B discusses the system-bath coupling.

\section{A. Isolated system dynamics}

We consider a harmonic oscillator with mass $m$ and timedependent frequency $\omega_{F}(t)$. The corresponding Hamiltonian is given by [37-43]

$$
H_{\mathrm{S}}^{F}(t)=\frac{p^{2}}{2 m}+\frac{1}{2} m \omega_{F}^{2}(t) x^{2},
$$

where the position and momentum operators $x$ and $p$ obey the canonical commutation relation $[x, p]=i \hbar$. We assume that the oscillator frequency $\omega_{F}(t)$ is modulated periodically with frequency $\omega_{L}$,

$$
\omega_{F}^{2}(t)=\omega_{0}^{2}\left[1+F \cos \left(\omega_{L} t+\phi_{L}\right)\right]
$$

where $F$ is the relative modulation amplitude. Throughout this paper we assume that $F=0$ for $t<0$. It follows that the Hamiltonian in Eq. (1) reduces to an ordinary harmonic oscillator with frequency $\omega_{0}$ and mass $m$ for negative times. In our model the driving amplitude is suddenly switched on at $t=0$ and held at a constant value for $t>0$. Note that the driving in Eq. (1) breaks the symmetry between position and momentum variables.

The time-dependent Schrödinger equation associated with the Hamiltonian in Eq. (1) can be solved [43-47],

$$
i \hbar \frac{\partial}{\partial_{t}} \psi_{n}(x, t)=H_{\mathrm{S}}^{F}(t) \psi_{n}(x, t) .
$$

The solutions $\psi_{n}(x, t)(n=0,1,2 \ldots)$ are described in Appendix $\mathrm{A}$ and reduce to the familiar harmonic oscillator states for $F=0$. It is possible to introduce generalized creation and annihilation operators that operate on the states $\psi_{n}(x, t)$ like in the undriven harmonic oscillator [31],

$$
\begin{aligned}
A(t)\left|\psi_{n}(t)\right\rangle & =\sqrt{n}\left|\psi_{n-1}(t)\right\rangle, \\
A^{\dagger}(t)\left|\psi_{n}(t)\right\rangle & =\sqrt{n+1}\left|\psi_{n+1}(t)\right\rangle .
\end{aligned}
$$

Note that the operators $A$ and $A^{\dagger}$ are time dependent.

Time-dependent Hamiltonians do generally not guarantee the validity of uncertainty relations and commutation relations [45]. However, the solution to the isolated system dynamics for our system Hamiltonian in Eq. (1) does not violate the uncertainty and commutation relations between canonical position and momentum operators [47].

\section{B. System-bath coupling}

We assume that the driven oscillator is weakly coupled to a heat bath of $N$ harmonic oscillators with the Hamiltonian,

$$
H_{B}=\sum_{r=1}^{N}\left(\frac{p_{r}^{2}}{2 m_{r}}+\frac{1}{2} m_{r} \omega_{r}^{2} x_{r}^{2}\right) .
$$

Here position and momentum operators of the $r$ th oscillator with mass $m_{r}$ and frequency $\omega_{r}$ are denoted by operators $x_{r}$ and $p_{r}$, respectively. The coupling between the bath and system is described by the Hamiltonian,

$$
H_{\mathrm{I}}^{\alpha}=-c_{\alpha} B,
$$

where the parameter $\alpha$ in the coupling operator,

$$
c_{\alpha}=\cos (\alpha) x+\sin (\alpha) \frac{p}{m \omega_{0}},
$$

describes which degrees of freedom are coupled to the bath. Note that $c_{\alpha=0}$ corresponds to position coupling, and $c_{\alpha=\pi / 2}$ realizes momentum coupling [48-51]. The bath operator $B$ in Eq. (6) is given by

$$
B=\sum_{r=1}^{N} \kappa_{r} x_{r},
$$

and $\kappa_{r}$ are coupling constants. The system-bath interaction Hamiltonian in Eq. (6) thus couples the system operator $c_{\alpha}$ to the position operators of the bath.

With the definitions in Eqs. (1), (5), and (6) we arrive at the total Hamiltonian for the driven oscillator coupled to the bath,

$$
H^{\alpha}=H_{S}^{F}(t)+H_{B}+H_{I}^{\alpha} .
$$


We assume the coupling to the bath to be weak and describe the quantum dynamics of the reduced density operator of the system $\rho$ by a Born-Markov master equation [29],

$$
\dot{\rho}=-\frac{i}{\hbar}\left[H_{\mathrm{S}}^{F}(t), \rho\right]+\mathcal{K} \rho,
$$

where the first term describes the coherent evolution and $\mathcal{K} \rho$ accounts for the interaction with the heat bath at temperature $T$,

$\mathcal{K} \rho=-\frac{1}{\hbar^{2}} \int_{0}^{\infty} d \tau \operatorname{Tr}_{B}\left\{\left[H_{\mathrm{I}}^{\alpha},\left[\tilde{H}_{\mathrm{I}}^{\alpha}(t-\tau, t), \rho_{B} \otimes \rho(t)\right]\right]\right\}$.

Here

$$
\rho_{B}=\exp \left(-\frac{H_{B}}{k_{\mathrm{B}} T}\right) / \operatorname{Tr}_{B}\left\{\exp \left(-\frac{H_{B}}{k_{\mathrm{B}} T}\right)\right\}
$$

is the density operator of the bath in thermal equilibrium and $k_{\mathrm{B}}$ is Boltzmann's constant. The operator $\tilde{H}_{\mathrm{I}}^{\alpha}$ in Eq. (11) is defined as

$$
\tilde{H}_{\mathrm{I}}^{\alpha}\left(t, t_{0}\right)=\left[U_{S}\left(t, t_{0}\right) U_{B}\left(t, t_{0}\right)\right]^{\dagger} H_{\mathrm{I}}^{\alpha}\left[U_{S}\left(t, t_{0}\right) U_{B}\left(t, t_{0}\right)\right],
$$

where $U_{B}\left(t, t_{0}\right)=\exp \left[-(i / \hbar) H_{B}\left(t-t_{0}\right)\right]$ describes the free evolution of the bath. The time evolution operator of the system is

$$
U_{S}\left(t, t_{0}\right)= \begin{cases}\mathcal{T}_{+} \exp \left(-\frac{i}{\hbar} \int_{t_{0}}^{t} d t^{\prime}\left[H_{\mathrm{S}}^{F}\left(t^{\prime}\right)\right]\right), & t \geqslant t_{0}, \\ \mathcal{T}_{-} \exp \left(\frac{i}{\hbar} \int_{t}^{t_{0}} d t^{\prime}\left[H_{\mathrm{S}}^{F}\left(t^{\prime}\right)\right]\right), & t<t_{0},\end{cases}
$$

where $\mathcal{T}_{+}$and $\mathcal{T}_{-}$are the chronological and antichronological time ordering operators, respectively.

Equation (11) can be evaluated for any system-bath interaction that justifies a Born-Markov approximation. In order to be specific, we assume that the heat bath exhibits an Ohmic spectral density with a Lorentz-Drude cutoff $\Omega \gg \omega_{0}$ [29], i.e.,

$$
J(\omega)=\frac{1}{\pi} \gamma \omega m \frac{\Omega^{2}}{\Omega^{2}+\omega^{2}},
$$

where $\gamma$ is the decay rate. This choice allows us to calculate the thermal correlation time of the bath explicitly and thus gives us full control over the validity of the Markov approximation. We find $\tau_{B}=\hbar /\left(2 \pi k_{B} T\right)$ (see Appendix B), and all considered temperatures $T$ in Sec. III are chosen such that $\tau_{B} \ll 1 / \gamma$ as required by the Markov approximation. With these assumptions Eq. (11) can be written as (see Appendix B)

$$
\begin{aligned}
\mathcal{K} \rho= & \frac{1}{\hbar} \int_{-\infty}^{\infty} d \omega J(\omega) n(\omega) \int_{0}^{\infty} d \tau e^{i \omega \tau}\left[\tilde{c}_{\alpha}(t-\tau, t) \rho, c_{\alpha}\right] \\
& + \text { H.c., }
\end{aligned}
$$

where H.c. is the Hermitian conjugate and

$$
n(\omega)=\frac{1}{e^{\hbar \omega / k_{B} T}-1}
$$

is the Bose-Einstein distribution. The operator $\tilde{c}_{\alpha}$ is defined as

$$
\tilde{c}_{\alpha}\left(t, t_{0}\right)=\left[U_{\mathrm{S}}\left(t, t_{0}\right)\right]^{\dagger} c_{\alpha} U_{\mathrm{S}}\left(t, t_{0}\right)
$$

and depends on the driving amplitude $F$ via the time evolution operator in Eq. (14).
In order to simplify the master equation further we take advantage of the fact that there exists a complete set of solutions to the isolated system dynamics. The details of the derivation are given in Appendix B. We find

$$
\begin{aligned}
\dot{\rho}= & -\frac{i}{\hbar}\left[H_{\mathrm{S}}^{F}(t), \rho\right]+\left\{\mathcal{S}_{1}^{\alpha}(t)\left[A(t) \rho A^{\dagger}(t)-A^{\dagger}(t) A(t) \rho\right]\right. \\
& +\mathcal{S}_{2}^{\alpha}(t)\left[A^{\dagger}(t) \rho A(t)-A(t) A^{\dagger}(t) \rho\right] \\
& +\mathcal{S}_{3}^{\alpha}(t)[A(t) \rho A(t)-A(t) A(t) \rho] \\
& \left.+\mathcal{S}_{4}^{\alpha}(t)\left[A^{\dagger}(t) \rho A^{\dagger}(t)-A^{\dagger}(t) A^{\dagger}(t) \rho\right]+\text { H.c. }\right\},
\end{aligned}
$$

where the time-dependent functions $\mathcal{S}_{i}^{\alpha}(i \in\{1,2,3,4\})$ are defined in Appendix B. In order to be consistent with the separation of timescales required by the Markov approximation [29,52,53], the definitions of $\mathcal{S}_{i}^{\alpha}(i \in\{1,2,3,4\})$ involve a time average to exclude any dynamic faster than the bath response time [see Eq. (B19)]. In this way we avoid tracking all the degeneracies in the Floquet eigenenergies [30,54].

We show in Sec. III that Eq. (19) can be approximated by a simpler master equation in the high-temperature regime, which we refer to as the high-temperature master equation (HTME). The HTME is obtained by ignoring the driving term in Eq. (18) which results in

$$
\tilde{c}_{\alpha}\left(t, t_{0}\right) \rightarrow e^{\frac{i}{\hbar} H_{\mathrm{S}}^{0}\left(t-t_{0}\right)} c_{\alpha} e^{-\frac{i}{\hbar} H_{\mathrm{S}}^{0}\left(t-t_{0}\right)} .
$$

With this additional approximation, the HTME can be written as

$$
\begin{aligned}
\dot{\rho}= & -\frac{i}{\hbar}\left[H_{\mathrm{S}}^{F}(t), \rho\right] \\
& +\left\{\frac{\gamma}{2} n\left(\omega_{0}\right)\left[a^{\dagger} \rho, a\right]+\frac{\gamma}{2}\left(n\left(\omega_{0}\right)+1\right)\left[a \rho, a^{\dagger}\right]\right. \\
& +\frac{\gamma}{2} n\left(\omega_{0}\right) e^{i 2 \alpha}\left[a^{\dagger} \rho, a^{\dagger}\right] \\
& \left.+\frac{\gamma}{2}\left(n\left(\omega_{0}\right)+1\right) e^{-i 2 \alpha}[a \rho, a]+\text { H.c. }\right\}
\end{aligned}
$$

where $a^{\dagger}$ and $a$ are the familiar creation and annihilation operators of the undriven harmonic oscillator.

\section{RESULTS}

Here we present a systematic study of the quasisteady state of the driven harmonic oscillator as a function of the external driving parameters, the temperature of the bath and the coupling angle $\alpha$ in the system-bath Hamiltonian. In a first step, we describe how we characterize the quasisteady state of the oscillator in Sec. III A. The numerical results are shown in Sec. III B.

We introduce dimensionless parameters which are frequently used to characterize periodically driven systems [55-57]. For the specific model at hand, $b=\left(2 \omega_{0} / \omega_{L}\right)^{2}$ is proportional to the ratio of natural and driving frequencies squared and $q=2 F \omega_{0}^{2} / \omega_{L}^{2}$ characterizes the effective driving strength. We further define $\zeta=\hbar \omega_{0} /\left(k_{B} T\right)$, which is proportional to the inverse temperature. In order to be consistent with the Born-Markov approximation, we set the decay rate to $\gamma / \omega_{0}=0.02$ and only consider parameters $\zeta \leqslant 3$ such that $\tau_{B} \gamma=\zeta \gamma /\left(2 \pi \omega_{0}\right) \ll 1$. Note that $\gamma$ enters the master equation (19) implicitly via the spectral density in Eq. (15). 


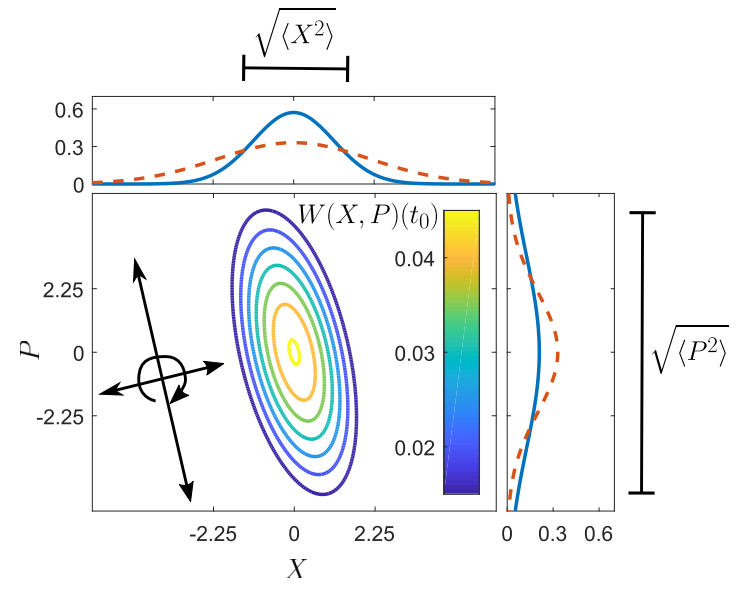

FIG. 2. The central panel shows a snapshot of $W(X, P)$ at time $t_{0} \gg 1 / \gamma$. The black arrow next to the ellipse indicates its dynamics consisting of simultaneous squeezing and rotation. The top and right panel contain the resulting probability distributions of the quadratures $X$ and $P$ (blue solid line). The red dashed line in the side panels corresponds to the initial thermal state for $t<0$. The width of the probability distribution is given by $\sqrt{\left\langle Y^{2}\right\rangle}$ with $Y=X, P$. Driving parameters are $b=0.1, q=0.5$, and $\zeta=0.7$ as defined in the text.

\section{A. Observables}

A descriptive way to illustrate the quantum state of the oscillator is given by the Wigner quasiprobability distribution defined as $[58,59]$

$$
W(X, P)(t)=\frac{1}{\pi} \int_{-\infty}^{\infty}\langle X+Y|\rho(t)| X-Y\rangle e^{-2 i P Y / \hbar} d Y .
$$

In this equation, the density operator $\rho(t)$ is determined by Eq. (19) and $X$ and $P$ are dimensionless position and momentum operators, respectively,

$$
\begin{aligned}
& X=\sqrt{\frac{m \omega_{0}}{\hbar} x,} \\
& P=\sqrt{\frac{1}{m \omega_{0} \hbar} p .}
\end{aligned}
$$

For $t<0$ the system is in a thermal state, which corresponds to a circular quasiprobability distribution with equally sized position and momentum quadratures $\left\langle X^{2}\right\rangle$ and $\left\langle P^{2}\right\rangle$, respectively. Once the driving is switched on at $t=0$, the shape of the Wigner function changes in time. A snapshot of $W(X, P)\left(t_{0}\right)$ at $t_{0} \gg 1 / \gamma$ is shown in Fig. 2. In contrast to the thermal distribution, $W(X, P)\left(t_{0}\right)$ has an elliptic shape with a tilted major axis. The value of $\left\langle X^{2}\right\rangle\left(\left\langle P^{2}\right\rangle\right)$ has decreased (increased) with respect to the thermal distribution. Even though this is just an example of the Wigner function at a particular point in time, the whole dynamics of $W(X, P)(t \gg 1 / \gamma)$ can be described by an ellipse rotating around the origin with period $4 \pi / \omega_{L}$. During one revolution the angular velocity of rotation changes in time, and the ellipse grows and shrinks along its major and minor axis.

The dynamics of $\left\{\left\langle X^{2}\right\rangle,\left\langle P^{2}\right\rangle,\langle X P+P X\rangle\right\}$ can be obtained from a closed set of equations since the total Hamil-

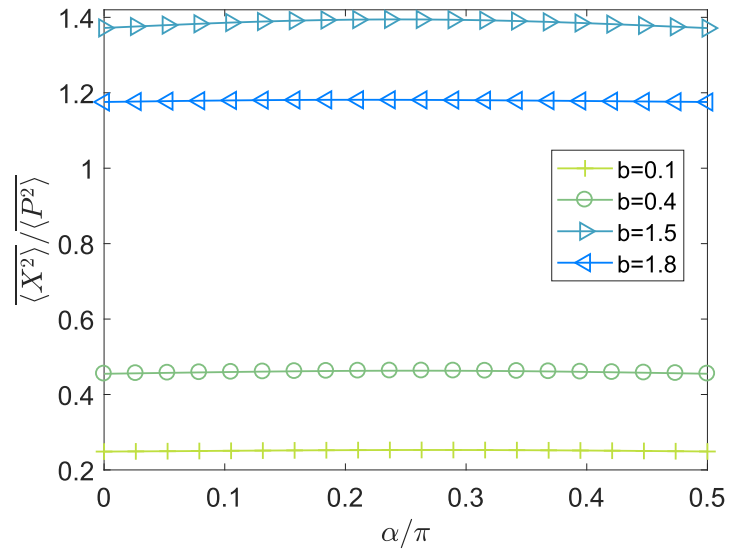

FIG. 3. Ratio of the time-averaged quadratures $\overline{\left\langle X^{2}\right\rangle} / \overline{\left\langle P^{2}\right\rangle}$ as a function of the coupling angle $\alpha$ and for different values of $b$. We have chosen $q=0.5, \zeta=10^{-4}$.

tonian $H^{\alpha}$ in Eq. (9) is quadratic and therefore preserves the Gaussian nature of the initial thermal state [60-65]. This set of equations is derived from the full master Eq. (19) and given in Appendix C. A similar closed set of equations can be obtained from the HTME in Eq. (21), and the corresponding results are denoted by $\langle\ldots\rangle_{\mathrm{HTME}}$. In the following we consider the steady-state regime $t \gg 1 / \gamma$ and restrict our analysis to $\overline{\left\langle X^{2}\right\rangle}$ and $\overline{\left\langle P^{2}\right\rangle}$, where the bar indicates a time average over the interval $\Delta t \approx 1 / \gamma \gg 2 \pi / \omega_{L}$. It follows that $\sqrt{\overline{\left\langle X^{2}\right\rangle}}$ and $\sqrt{\overline{\left\langle P^{2}\right\rangle}}$ characterize the width of the time-averaged Wigner function in $X$ and $P$, respectively.

\section{B. Numerical results}

Here we systematically investigate the dependence of $\overline{\left\langle X^{2}\right\rangle}$ and $\overline{\left\langle P^{2}\right\rangle}$ on the parameters $b, \zeta$, and $\alpha$. In a first step, we find that the ratio $\overline{\left\langle X^{2}\right\rangle} / \overline{\left\langle P^{2}\right\rangle}$ is approximately independent of the coupling angle $\alpha$ as shown in Fig. 3. This ratio only depends on the driving frequency and strength, and is larger (smaller) than unity for $b>1(b<1)$. The shape of the time-averaged Wigner function thus deviates significantly from a circle in the presence of the parametric driving. The results in Fig. 3 correspond to the high-temperature limit $\zeta=10^{-4}$. However, we find that the ratio $\overline{\left\langle X^{2}\right\rangle} / \overline{\left\langle P^{2}\right\rangle}$ is also approximately independent of $\zeta$ for the considered range of values $\zeta \in\left[10^{-4}, 3\right]$.

The previously discussed dependence of $\overline{\left\langle X^{2}\right\rangle}$ and $\overline{\left\langle P^{2}\right\rangle}$ allows us to restrict the following analysis to one of the two quadratures. We choose $\overline{\left\langle X^{2}\right\rangle}$ and investigate its dependence on $b, \alpha$, and $\zeta$ by introducing the short-hand notation,

$$
R_{X}=\overline{\left\langle X^{2}\right\rangle} /\left\langle X^{2}\right\rangle_{\text {thermal }},
$$

where $\left\langle X^{2}\right\rangle_{\text {thermal }}$ is the undriven initial thermal value of $\overline{\left\langle X^{2}\right\rangle}$. A value of $R_{X}>1\left[R_{X}<1\right]$ thus means that the driving enhances (reduces) the value of $\overline{\left\langle X^{2}\right\rangle}$ compared to its thermal value.

The dependence of $R_{X}$ on $\zeta$ is shown in Figs. 4(a) and 4(b) for position and momentum coupling, respectively. Note that all values for $R_{X}$ in Fig. 4 differ from unity and hence the parametric driving changes the value of $\overline{\left\langle X^{2}\right\rangle}$ compared 

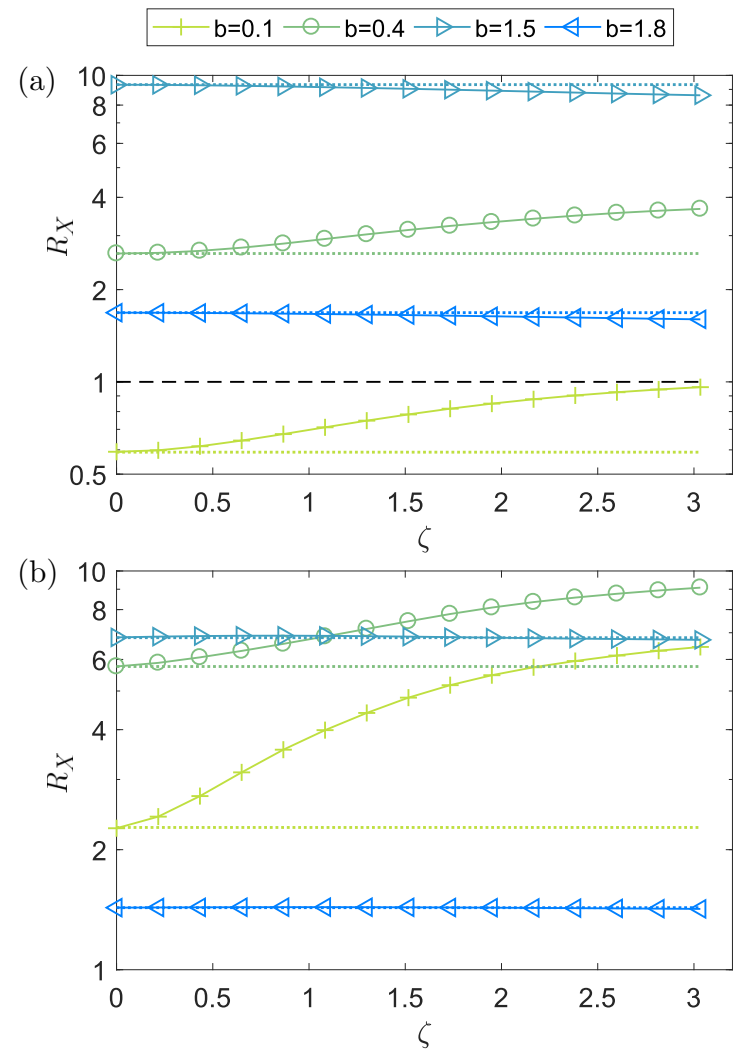

FIG. 4. $R_{X}$ as a function of inverse temperature $\zeta$ for $q=0.5$, different driving frequencies, and coupling angle (a) $\alpha=0$ and (b) $\alpha=\frac{\pi}{2}$. Dotted lines of the same color indicate the results obtained from the evolution of the HTME. Dashed line at $R_{X}=1$ in (a) is a guide to the eye.

to $\left\langle X^{2}\right\rangle_{\text {thermal }}$. Furthermore, we note that the results for $R_{X}$ in Fig. 4 differ significantly for position and momentum coupling. Most prominently, $R_{X}>1$ for all curves in Fig. 4(b) (momentum coupling), while $R_{X}<1$ for position coupling and $b=0.1$. The reduction of $\left(R_{X}\right)_{\zeta \rightarrow 0}$ below unity for position coupling and fast driving $(b \ll 1)$ has already been studied in Refs. [60,66-68]. In general, we find that $R_{X}$ strongly increases with $\zeta$ for both coupling types if $b<1$. On the other hand, $R_{X}$ shows a much weaker dependence on $\zeta$ for $b>1$. The value of $\left(R_{X}\right)_{b>1}$ slightly decreases with $\zeta$ for position coupling and remains approximately constant for momentum coupling.

In order to illustrate the significance of the $\zeta$ dependence of $R_{X}$, we show the results for $\left(R_{X}\right)_{\text {HTME }}$ obtained via the HTME in Eq. (21) by the horizontal dotted lines in Fig. 4. Since they exhibit no $\zeta$ dependence, the temperature dependence of $R_{X}$ predicted by the full master equation is a direct consequence of the parametric driving on the system-bath coupling. The differences between the HTME and full master equation are most pronounced near $\zeta=3$ and for $b=0.1$. On the other hand, we find that $R_{X}$ converges to $\left(R_{X}\right)_{\mathrm{HTME}}$ in the limit $\zeta \rightarrow 0$ which can be understood as follows. The thermal correlation time of the bath $\tau_{B}=\hbar /\left(2 \pi k_{B} T\right)[29,53]$ becomes much smaller than the typical timescale $\tau_{\mathrm{S}}=\min \left(1 / \omega_{0}, 1 / \omega_{L}\right)$ of the system dynamics in the high-temperature limit. Consequently, the time evolution of the system cannot influence the system-bath coupling.

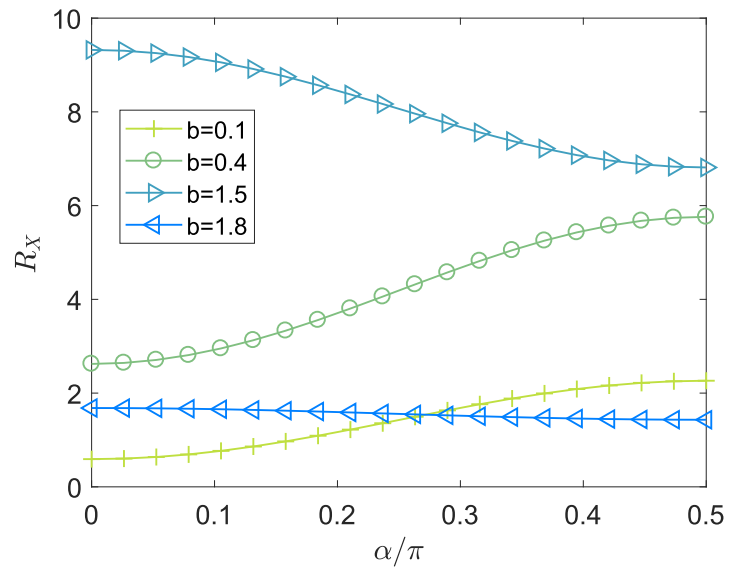

FIG. 5. $R_{X}$ as a function of coupling angle $\alpha$ for different values of $b$, driving strength $q=0.5$, and inverse temperature $\zeta=10^{-4}$.

Next we investigate the $\alpha$ dependence of $R_{X}$ in the hightemperature limit and for different driving frequencies. The results are shown in Fig. 5. We observe an increase (decrease) of $R_{X}$ with increasing $\alpha$ for $b<1(b>1)$. This behavior is similar to the functional dependence of $R_{X}$ on $\zeta$ and $b$ shown in Fig. 4. We thus find that increasing $\alpha$ or $\zeta$ leads to qualitatively comparable effects. Most importantly, $R_{X}$ shows a strong dependence on $\alpha$ for $b=0.4$ and $b=1.5$. Since $R_{X}$ is monotonously increasing (decreasing) for $b=0.4(b=1.5)$, one can determine the coupling angle $\alpha$ by comparing $\overline{\left\langle X^{2}\right\rangle}$ to $\left\langle X^{2}\right\rangle_{\text {thermal }}$. Note that the $\alpha$ dependence of the results in Fig. 5 is a direct consequence of the counter-rotating terms $\propto e^{ \pm 2 i \alpha}$ in the HTME in Eq. (21).

In order to identify the parameter regime with the strongest $\alpha$ dependence, we move away from the high-temperature limit and show $R_{X}$ as a function of $b$ for different values of $\alpha$ in Fig. 6 . Here the left and right panels correspond to the parameter range $b<0.5$ and $b>1.45$, respectively. For values closer to $b=1$ the solutions become unstable and no steady state can be achieved. Figure 6 shows that $R_{X}$ exhibits the strongest
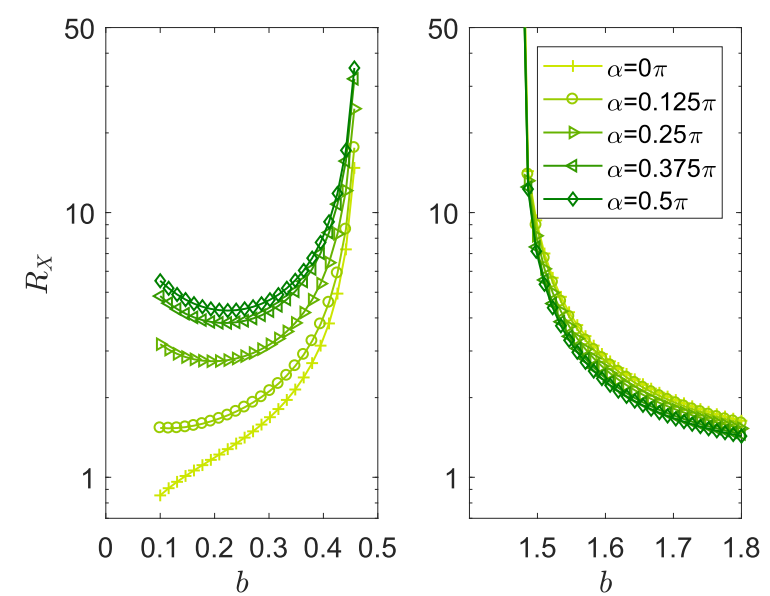

FIG. 6. $R_{X}$ as a function of inverse driving frequencies for different coupling angles $\alpha$, driving strength $q=0.5$, and inverse temperature $\zeta=2$. The left and right panel correspond to driving above and below the resonance frequency, respectively. 


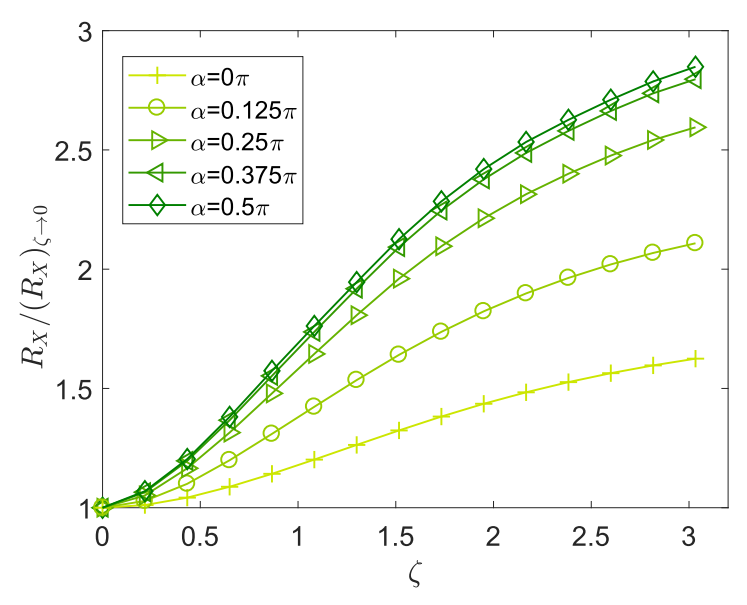

FIG. 7. $R_{X} /\left(R_{X}\right)_{\zeta \rightarrow 0}$ as a function of $\zeta$ for $b=0.1, q=0.5$, and different values of $\alpha$. The values of $\left(R_{X}\right)_{\zeta \rightarrow 0}$ can be obtained from the $b=0.1$ curve in Fig. 5 .

dependence on $\alpha$ for $b \approx 0.1$. In particular, note that the range of the $R_{X}$ values from $\alpha=0$ to $\alpha=\pi / 2$ is larger than in the corresponding curve for $b=0.1$ in Fig. 5 due to the different $\zeta$ values.

Finally, we focus on $b=0.1$ and investigate the $\zeta$ dependence of $R_{X}$ for different values of $\alpha$. We find that $R_{X}$ increases relative to its $\zeta \rightarrow 0$ value for all considered values of $\alpha$ as shown in Fig. 7. The relative increase with $\zeta$ is the largest for $\alpha=\pi / 2$ and the smallest for $\alpha=0$. Furthermore, we find that small changes in $\alpha$ change the $\zeta$ dependence of $R_{X} /\left(R_{X}\right)_{\zeta \rightarrow 0}$ most significantly near $\alpha=0$.

Two conclusions can be drawn from the results in Fig. 7. First, increasing the value of $\zeta$ increases the spread of $R_{X}$ with $\alpha$. It thus becomes easier to determine $\alpha$ via a comparison of $\overline{\left\langle X^{2}\right\rangle}$ and $\left\langle X^{2}\right\rangle_{\text {thermal }}$ for smaller temperatures of the bath. Second, the difference of the $\zeta$ dependence of $R_{X}$ for various choices of $\alpha$ opens up a second route to determining $\alpha$. For example, one could increase $\zeta$ by a given factor and measure the corresponding increase in $R_{X}$. According to Fig. 7, this increase is a unique function of $\alpha$. Note that this approach works best for small $\alpha$ where the $\zeta$ dependence of $R_{X}$ is most sensitive to changes in $\alpha$.

\section{DISCUSSION OF $\alpha$-DEPENDENCE}

The strong $\alpha$ dependence of the results in Sec. III is a direct consequence of the driving. In order to see this, we note that the full master Eq. (19) reduces to the HTME in Eq. (21) in the case of an undriven system $(F=0)$. Since only the counter-rotating terms exhibit an $\alpha$ dependence, all $\alpha$-dependent terms are of the order of $\gamma / \omega_{0}$ for $F=0$. In the regime of weak system-bath coupling $\gamma / \omega_{0} \ll 1$ considered here, the $\alpha$ dependence is negligibly small. It is thus justified to perform a rotating-wave approximation [29] in the HTME which completely removes the $\alpha$ dependence and reduces the HTME to the standard quantum optical master equation. With this approximation, the density operator and hence all expectation values are independent of $\alpha$ in the undriven regime.

Note that the approximate $\alpha$ independence in the absence of the driving is not only a feature of our quantum model described in Sec. II, but extends to the corresponding classical model if $\gamma / \omega_{0} \ll 1$. The equations of motion for the classical, dimensionless position, and momentum variables $\mathcal{X}$ and $\mathcal{P}$ for $F=0$ are

$$
\begin{aligned}
& \frac{d \mathcal{X}}{d \tau}=-\frac{\gamma}{\omega_{0}} \sin ^{2}(\alpha) \mathcal{X}+\mathcal{P}\left(1+\frac{\gamma}{2 \omega_{0}} \sin (2 \alpha)\right), \\
& \frac{d \mathcal{P}}{d \tau}=-\frac{\gamma}{\omega_{0}} \cos ^{2}(\alpha) \mathcal{P}-\mathcal{X}\left(1-\frac{\gamma}{2 \omega_{0}} \sin (2 \alpha)\right),
\end{aligned}
$$

where we ignored noise terms and introduced $\tau=t \omega_{0}$. These equations coincide with the equations of motion for $\langle X\rangle$ and $\langle P\rangle$ derived from the HTME, and all $\alpha$-dependent terms are proportional to $\gamma / \omega_{0}$. The classical motion thus only exhibits a significant $\alpha$ dependence for extremely strong damping of the order of $\gamma \approx \omega_{0}$, which is outside the parameter range considered here.

Having established that the strong $\alpha$ dependence of our results is a consequence of the driving, we now investigate its origin in more detail. According to [27], our results are a manifestation of EGD which can be explained in terms of quasidegeneracies of the Floquet eigenenergies of the quantum problem. Due to the infinite number of Floquet eigenenergies in our problem, the aim of this section is to give an alternative and more intuitive picture for the strong $\alpha$ dependence of our results. To this end we show in Appendix D that the total Hamiltonian $H^{\alpha}$ in Eq. (9) is unitarily equivalent to

$$
\mathcal{H}^{\alpha}=H_{\mathrm{S}}^{F}(t)+H_{\mathrm{B}}+\mathcal{H}_{\mathrm{I}}^{\alpha}+H_{\text {shift }},
$$

where $H_{\mathrm{S}}^{F}(t)$ and $H_{\mathrm{B}}$ remain unchanged and $H_{\text {shift }}$ is given in Appendix D. The latter term describes small shifts of the bath and system frequencies and will be neglected in the following. The transformed interaction Hamiltonian $\mathcal{H}_{\mathrm{I}}^{\alpha}$ is given by

$$
\mathcal{H}_{\mathrm{I}}^{\alpha}=-x B^{\alpha},
$$

where

$$
B^{\alpha}=\sum_{r=1}^{N} \kappa_{r}\left(\cos (\alpha) x_{r}-\frac{\sin (\alpha)}{m_{r} \omega_{0}} p_{r}\right) .
$$

In contrast to $H_{\mathrm{I}}^{\alpha}$ in Eq. (6), the system-bath coupling in $\mathcal{H}_{\mathrm{I}}^{\alpha}$ is mediated by the position coordinate of the oscillator coupled to an $\alpha$-dependent superposition of position and momentum operators of the bath modes. In this way, the $\alpha$ dependence has been entirely moved from the coupling operator to the bath.

Following the steps in Appendix B we derive a master equation for the density operator $\rho_{U}$ from the transformed Hamiltonian $\mathcal{H}^{\alpha}$; see Appendix D. We have explicitly verified that $\rho_{U}$ and $\rho$ lead to the same results for all parameters investigated in Sec. IIIB. In the derivation of the master equation for $\rho_{U}$, the linear superposition of bath operators in Eq. (28) gives rise to an $\alpha$-dependent spectral density,

$$
J^{\alpha}(\omega)=J(\omega)\left[\cos (\alpha)^{2}+\left(\frac{\omega}{\omega_{0}}\right)^{2} \sin ^{2}(\alpha)\right],
$$

which is the product of the Ohmic spectral density $J(\omega)$ in Eq. (15) and an $\alpha$-dependent function. We show $J^{\alpha}(\omega)$ for the two extreme cases $\alpha=0$ and $\alpha=\pi / 2$ in Fig. 8. While $J^{0}(\omega)$ and $J^{\pi / 2}(\omega)$ are identical at $\omega= \pm \omega_{0}$, they differ significantly for $|\omega| \neq \omega_{0}$. 


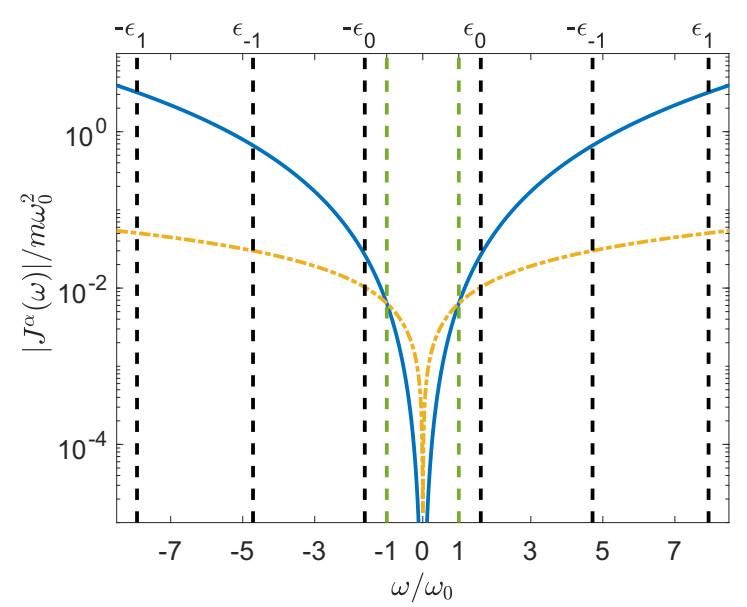

FIG. 8. The dash-dotted yellow (solid blue) line corresponds to the bath spectral density $J^{\alpha=0}(\omega)\left[J^{\alpha=\pi / 2}(\omega)\right]$. The green dashed vertical lines mark the undriven system frequency $\pm \omega_{0}$. The scaled Floquet quasifrequencies $\epsilon_{r}=\left(\mu+r \omega_{L}\right) / \omega_{0}$ for $q=0.5$ and $b=$ 0.1 are indicated by black dashed vertical lines.

We are now in a position to understand the $\alpha$ dependence of the results in Sec. III B. In general, the system-bath interaction is determined by the values of $J^{\alpha}\left(\omega_{c}\right)$ at the characteristic frequencies $\omega_{c}$ of the classical solution of the undamped harmonic oscillator (see Appendix E for details). We have found that all observables in Sec. IIIB are independent of $\alpha$ for an undriven oscillator. In this case, the resonance frequency $\omega_{0}$ is the only characteristic frequency involved in the system dynamics. However, the spectral density in Eq. (29) satisfies $J^{\alpha}\left( \pm \omega_{0}\right)=J\left( \pm \omega_{0}\right)$ for all $\alpha$, and hence the system-bath interaction is independent of $\alpha$.

This picture changes significantly if the driving is switched on. The driving leads to new characteristic frequencies $\omega_{c}=$ $\pm \mu+r \omega_{L}$, with $r \in \mathbb{Z}$ and where $\mu$ is the quasifrequency of the Floquet spectrum closest to $\omega_{0}$ of the underlying classical model of a driven but undamped harmonic oscillator. Since the spectral density $J^{\alpha}$ depends strongly on $\alpha$ for $|\omega|>\omega_{0}$ (see Fig. 8), the parametric driving leads to $\alpha$-dependent results.

\section{SUMMARY AND CONCLUSION}

We have shown that parametric driving of a harmonic oscillator coupled to a heat bath allows one to distinguish between different microscopic models for the system-bath interaction. More specifically, we have considered a bath with an Ohmic spectral density that couples to a linear superposition of the position and momentum degrees of freedom of the oscillator. This superposition is parametrized via the coupling angle $\alpha$ which allows one to continuously change the character of the coupling from position to momentum coupling. We have systematically investigated the dependence of the timeaveraged expectation values of $\overline{\left\langle X^{2}\right\rangle}$ and $\overline{\left\langle P^{2}\right\rangle}$ on the driving parameters, temperature $T$ of the bath, and $\alpha$. While $\overline{\left\langle X^{2}\right\rangle} / \overline{\left\langle P^{2}\right\rangle}$ is approximately independent of $\alpha$ and $T$, we have shown that $R_{X}=\overline{\left\langle X^{2}\right\rangle} /\left\langle X^{2}\right\rangle_{\text {thermal }}$ shows a strong dependence on temperature and on the coupling angle $\alpha$. This dependence on the coupling angle could be used to determine $\alpha$ by measuring $\overline{\left\langle X^{2}\right\rangle}\left(\left\langle X^{2}\right\rangle_{\text {thermal }}\right.$ in the presence (absence) of the parametric driving. We have found that $R_{X}$ exhibits the strongest dependence on $\alpha$ for large driving frequencies $\omega_{L} \gg \omega_{0}$ and for bath temperatures with $k_{B} T \leqslant \hbar \omega_{0}$. In this regime, $R_{X}$ also displays a characteristic dependence on inverse temperature for each value of $\alpha$, thus offering an additional route to determine the value of $\alpha$ in the interaction Hamiltonian.

Our results have been obtained within the framework of a master equation in the Born-Markov approximation that accounts for the modification of the system-bath interaction due to the parametric driving. In order to be consistent with the Markov approximation, we describe the time evolution of the reduced density operator of the oscillator on a coarse-grained time axis with resolution $\Delta t$, where $\Delta t$ is much smaller than $1 / \gamma$ and much larger than the bath correlation time. We compare our results to a simpler master equation that neglects the influence of the driving on the dissipative part and where all counter-rotating terms are kept. We find that this HTME agrees with the full master equation in the high-temperature limit where $\hbar \omega_{0} \ll k_{B} T$, but deviates significantly otherwise. We thus conclude that the influence of the parametric driving on the system-bath coupling must be taken into account in general.

The $\alpha$ dependence of our results can be understood within the general framework of EGD introduced in [27], where the dependence of the system dynamics on the coupling operator is explained in terms of quasidegeneracies of the Floquet spectrum. In addition, we have provided a complementary and intuitive explanation of the $\alpha$ dependence of our results by applying a unitary transformation to the total system-bath Hamiltonian. In this transformed picture, the position variable of the oscillator couples to an $\alpha$-dependent superposition of bath variables which results in an $\alpha$-dependent spectral density $J^{\alpha}$. Since the system-bath coupling is determined by the values of $J^{\alpha}$ at the Floquet quasifrequencies associated with the parametric driving, the density operator obtained from this master equation depends on $\alpha$ if the system is driven.

Our results open up several directions for future investigations. The explanation of the $\alpha$ dependence of our results via the unitarily equivalent model giving rise to an $\alpha$-dependent spectral density shows that strong parametric driving allows one to probe an unknown spectral density if the microscopic coupling mechanism between the system and the bath is known. Furthermore, this approach could be extended to investigate systems with several competing dissipation mechanisms or to the nonMarkovian regime of strong damping which is often encountered in solid-state materials. This could either be achieved by using the Feynman-Vernon path integral formalism [60] or the stochastic Liouville-von Neumann equation [69]. Eventually, an improved understanding of system-bath interactions in these systems paves the way to control and engineer their quantum dynamics via strong driving. For example, cooling of a driven harmonic oscillator coupled to a non-Markovian bath can be achieved via optimal control techniques $[65,70]$. This could allow one to prepare the oscillator in nonclassical, squeezed states [71-74]. 


\section{ACKNOWLEDGMENTS}

M.K. thanks the National Research Foundation and the Ministry of Education of Singapore for support. The research leading to these results has received funding from the European Research Council under the European Union's Seventh Framework Programme (FP7/2007-2013)/ERC Grant Agreement No. 319286 Q-MAC and from EPSRC programme Grant No. EP/P009565/1 DesOEQ.

\section{APPENDIX A: SOLUTION OF THE DRIVEN HARMONIC OSCILLATOR}

Here we describe the solutions $\psi_{n}(x, t)$ to the timedependent Schrödinger equation in Eq. (3) following the approach in [46,47] that is based on dynamical invariants $[41,75]$. We write the solutions as

$$
\begin{aligned}
\psi_{n}(x, t)= & \frac{1}{\sqrt{2^{n} n !}}\left(\frac{m \omega_{I}}{\pi \hbar g_{-}(t)}\right)^{\frac{1}{4}} e^{-i \frac{g_{0}(t)}{2 \hbar g_{-}(t)} x^{2}} e^{-i \Lambda(t)\left(n+\frac{1}{2}\right)} \\
& \times e^{-\frac{m \omega_{I}}{2 \hbar g_{-}(t)} x^{2}} H_{n}\left(\sqrt{\frac{m \omega_{I}}{\hbar g_{-}(t)}} x\right)
\end{aligned}
$$

where

$$
\Lambda(t)=\int_{0}^{t} d t^{\prime} \frac{\omega_{I}}{g_{-}\left(t^{\prime}\right)}
$$

is a global phase,

$$
\omega_{I}=\frac{1}{m} \sqrt{g_{+}(t) g_{-}(t)-g_{0}^{2}(t)}
$$

is a time-independent constant frequency, and $H_{n}$ are the Hermite polynomials of degree $n$. The functions $g_{+}(t), g_{+}(t)$, and $g_{+}(t)$ in Eq. (A1) are defined as

$$
\begin{aligned}
g_{+}(t) & =m^{2} \dot{f}_{1}(t) \dot{f}_{2}(t), \\
g_{0}(t) & =-\frac{m}{2}\left[\dot{f}_{1}(t) f_{2}(t)+f_{1}(t) \dot{f}_{2}(t)\right], \\
g_{-}(t) & =f_{1}(t) f_{2}(t),
\end{aligned}
$$

where $f_{i}(t)(i \in\{1,2\})$ are two linearly independent solutions of the classical harmonic oscillator. These functions obey

$$
\ddot{f}_{i}(t)+\omega^{2}(t) f_{i}(t)=0
$$

and we impose the initial condition $f_{i}(0)=1$ at $t=0$ and require $f_{1}(t)=f_{2}(t)^{*}$. For $t<0$ where the driving is absent we thus find $f_{1,2}(t<0)=e^{ \pm i \omega_{0} t}$, and consequently $g_{0}(t<$ $0)=0$. Furthermore, as $g_{-}(t<0)=1$ the chosen initial conditions result in $\omega_{I}=\omega_{0}$ such that $\psi_{n}(x, t)$ reduces to the harmonic oscillator eigenstates. These states form an orthogonal basis and the states $\psi_{n}(x, t)$ remain orthogonal for $t \geqslant 0$, i.e., $\left\langle\psi_{n}(t) \mid \psi_{m}(t)\right\rangle=\delta_{n, m}$ as the time dependence of the argument $\frac{m \omega_{I}}{\hbar g_{-}(t)}$ of the integral in the equal time norm can be eliminated by a change of variables. It follows that the states $\left|\psi_{n}(t)\right\rangle$ in Eq. (A1) span a complete basis at all times.

We define generalized creation and annihilation operators $A^{\dagger}$ and $A$ that act on the states $\left|\psi_{n}(t)\right\rangle$ as described in Eq. (4). The annihilation operator is given by [31]

$$
A(t)=\frac{1}{\sqrt{2}}\left[\sqrt{\frac{m \omega_{0}}{\hbar}} h_{1}(t) x+\sqrt{\frac{1}{\hbar m \omega_{0}}} h_{2}(t) p\right],
$$

and the complex functions $h_{1}(t)$ and $h_{2}(t)$ are defined as

$$
\begin{aligned}
& h_{1}(t)=\exp (i \Lambda(t)) \sqrt{\frac{\omega_{I}}{\omega_{0} g_{-}(t)}}\left(1+i \frac{g_{0}(t)}{m \omega_{I}}\right), \\
& h_{2}(t)=i \exp (i \Lambda(t)) \sqrt{\frac{\omega_{0} g_{-}(t)}{\omega_{I}}} .
\end{aligned}
$$

Since $[x, p]=i \hbar$ and $h_{1}(t) h_{2}^{*}(t)-h_{1}^{*}(t) h_{2}(t)=-2 i$, the equal-time commutation relation $\left[A(t), A^{\dagger}(t)\right]=1$ is satisfied.

\section{APPENDIX B: MASTER EQUATION DERIVATION}

Here we discuss the derivation of the master equation in Sec. II B. Our approach builds on the work presented in [31], but considers a more general system-bath coupling, does not use the Floquet basis states, and employs the weakest possible rotating-wave approximation in order to be consistent with the Born-Markov approximation.

In a first step we rewrite the dissipative term $\mathcal{K} \rho$ in Eq. (11) as follows [29,30,54],

$$
\begin{aligned}
\mathcal{K} \rho= & \frac{1}{\hbar^{2}} \int_{0}^{\infty} d \tau\left(\frac{i}{2} D(\tau)\left[c_{\alpha},\left\{\tilde{c}_{\alpha}(t-\tau, t), \rho\right\}\right]\right. \\
& \left.-\frac{1}{2} D_{1}(\tau)\left[c_{\alpha},\left[\tilde{c}_{\alpha}(t-\tau, t), \rho\right]\right]\right),
\end{aligned}
$$

where $\{Q, W\}=Q W+W Q$ is the anticommutator for operators $Q, W$ and we introduced following [29] the dissipation kernel,

$$
D(\tau)=i\langle[B, \tilde{B}(-\tau)]\rangle=i[B, \tilde{B}(-\tau)],
$$

and the noise kernel,

$$
D_{1}(\tau)=\langle\{B, \tilde{B}(-\tau)\}\rangle .
$$

We find

$$
\begin{aligned}
D(\tau) & =2 \hbar \int_{0}^{\infty} d \omega J(\omega) \sin \omega \tau, \\
D_{1}(\tau) & =2 \hbar \int_{0}^{\infty} d \omega J(\omega) \operatorname{coth}\left(\hbar \omega / 2 k_{B} T\right) \cos \omega \tau,
\end{aligned}
$$

and

$$
J(\omega)=\sum_{n=1}^{N} \frac{\kappa_{n}^{2}}{2 m_{n} \omega_{n}} \delta\left(\omega-\omega_{n}\right)
$$

is the spectral density. As described in the main text we assume that $J(\omega)$ is Ohmic with a Lorentz-Drude cutoff as given in Eq. (15). The longest thermal correlation time is then given by [29] $\tau_{B}=\max \left[\hbar /\left(2 \pi k_{B} T\right), \Omega^{-1}\right]$. We set $\Omega=10^{5} \omega_{0}$ such that for all considered parameters $\tau_{B}=\hbar /\left(2 \pi k_{B} T\right)$. With the identities $J(\omega)=-J(-\omega)$ as well as $-n(-\omega)=n(\omega)+1$ we obtain Eq. (16).

The solution of the master Eq. (10) with $\mathcal{K} \rho$ as in Eq. (16) is greatly simplified by projecting it onto the states $\left|\psi_{n}(t)\right\rangle$ which solve the full Schrödinger equation of the driven oscillator. The matrix elements of the density operator of the system in this basis are denoted by

$$
\rho_{n m}(t)=\left\langle\psi_{n}(t)|\rho(t)| \psi_{m}(t)\right\rangle,
$$


and evolve only due to the system-bath coupling. We find

$$
\begin{aligned}
\dot{\rho}_{n m}(t)= & \sum_{k, l}\left[\bar{C}_{n k}^{\alpha}(t) \rho_{k l}(t) C_{l m}^{\alpha}(t)\right. \\
& \left.-C_{n k}^{\alpha}(t) \bar{C}_{k l}^{\alpha}(t) \rho_{l m}(t)\right]+ \text { H.c. },
\end{aligned}
$$

where

$$
\begin{aligned}
C_{n m}^{\alpha}(t)= & \left\langle\psi_{n}(t)\left|c_{\alpha}\right| \psi_{m}(t)\right\rangle, \\
\bar{C}_{n m}^{\alpha}(t)= & \frac{1}{\hbar} \int_{-\infty}^{\infty} d \omega J(\omega) n(\omega) \\
& \times \int_{0}^{\infty} d \tau e^{i \omega \tau} C_{n m}^{\alpha}(t-\tau) .
\end{aligned}
$$

In order to evaluate these matrix elements, we express the coupling operator $c_{\alpha}$ in Eq. (7) in terms of the generalized creation and annihilation operators $A(t)$ and $A^{\dagger}(t)$,

$$
c_{\alpha}=s_{1}^{\alpha}(t) A^{\dagger}(t)+s_{2}^{\alpha}(t) A(t)
$$

where

$$
s_{1}^{\alpha}(t)=i \sqrt{\frac{\hbar}{2 m \omega_{0}}}\left[\sin (\alpha) h_{1}(t)-\cos (\alpha) h_{2}(t)\right]
$$

is a complex-valued function and $s_{2}^{\alpha}(t)=\left[s_{1}^{\alpha}(t)\right]^{*}$. The matrix element in Eq. (B8a) thus reads

$$
C_{n m}^{\alpha}(t)=s_{1}^{\alpha}(t) A_{n m}^{\dagger}+s_{2}^{\alpha}(t) A_{n m}
$$

and the time-independent matrix elements of the creation and annihilation operators are given by

$$
\begin{aligned}
& A_{n m}^{\dagger}=\left\langle\psi_{n}(t)\left|A^{\dagger}(t)\right| \psi_{m}(t)\right\rangle=\sqrt{m+1} \delta_{n, m+1}, \\
& A_{n m}=\left\langle\psi_{n}(t)|A(t)| \psi_{m}(t)\right\rangle=\sqrt{m} \delta_{n, m-1} .
\end{aligned}
$$

Next we discuss the evaluation of $\bar{C}_{n m}$ in Eq. (B8b).

With the help of Eq. (B11), we find

$$
\bar{C}_{n m}^{\alpha}(t)=\bar{s}_{1}^{\alpha}(t) A_{n m}^{\dagger}+\bar{s}_{2}^{\alpha}(t) A_{n m}
$$

where

$$
\bar{s}_{i}^{\alpha}(t)=\frac{1}{\hbar} \int_{-\infty}^{\infty} d \omega J(\omega) n(\omega) \int_{0}^{\infty} d \tau e^{i \omega \tau} s_{i}^{\alpha}(t-\tau) .
$$

In order to evaluate Eq. (B14), we choose a sufficiently large time interval $\left[t_{i}, t_{f}\right]$ of length $T_{i f}=t_{f}-t_{i}$ to avoid artefacts from the Gibbs effect [76] and represent $s_{i}^{\alpha}$ in terms of the first $N_{k}$ terms of its discrete Fourier series,

$$
s_{i}^{\alpha}\left(t_{i}<t<t_{f}\right) \approx \sum_{k=-k_{\max }}^{k_{\max }} \mathcal{F}\left[s_{i}^{\alpha}\right]\left(\omega_{k}\right) e^{i w_{k} t}
$$

Here $w_{k}=\frac{2 \pi k}{T_{i f}}$ are the discrete frequencies with integer index $k, k_{\max }=\frac{N_{k}-1}{2}$, and the Fourier coefficients $\mathcal{F}\left[s_{i}^{\alpha}\right]\left(\omega_{k}\right)$ are defined as

$$
\mathcal{F}\left[s_{i}^{\alpha}\right]\left(\omega_{k}\right)=\frac{1}{T_{i f}} \int_{t_{i}}^{t_{f}} s_{i}^{\alpha}(t) e^{-i w_{k} t} d t
$$

The expansion in Eq. (B15) allows us to evaluate the integrals in Eq. (B14). We find

$$
\bar{s}_{i}^{\alpha}(t)=\frac{\pi}{\hbar} \sum_{k=-k_{\max }}^{k_{\max }} J\left(w_{k}\right) n\left(w_{k}\right) e^{i w_{k} t} \mathcal{F}\left[s_{i}^{\alpha}\right]\left(\omega_{k}\right),
$$

where we employed the identity $\int_{0}^{\infty} d t e^{i \omega t}=\pi \delta(\omega)+P(i / \omega)$ and neglected the principal part.

We now return to the master Eq. (B7). Substituting Eqs. (B11) and (B13) in Eq. (B7) results in a differential equation for $\rho_{n m}$ with time-dependent coefficients $s_{i}^{\alpha}(t) \bar{s}_{j}^{\alpha}(t)$. In order to be consistent with the Born-Markov approximation we average these time-dependent terms over the correlation time of the bath $\tau_{B}$ times a constant factor $f$. In order to make sure that the bath cannot introduce any dynamics faster than $\tau_{B}$, we set $f=10$ in Eq. (B19). This is necessary since the Markov approximation assumes that the system does not evolve appreciably over this timescale. We find

$$
\begin{aligned}
\dot{\rho}_{n m}(t)= & \sum_{k, l}\left\{\mathcal{S}_{1}^{\alpha}(t)\left[A_{n k} \rho_{k l} A_{l m}^{\dagger}-A_{n k}^{\dagger} A_{k l} \rho_{l m}\right]\right. \\
& +\mathcal{S}_{2}^{\alpha}(t)\left[A_{n k}^{\dagger} \rho_{k l} A_{l m}-A_{n k} A_{k l}^{\dagger} \rho_{l m}\right] \\
& +\mathcal{S}_{3}^{\alpha}(t)\left[A_{n k} \rho_{k l} A_{l m}-A_{n k} A_{k l} \rho_{l m}\right] \\
& \left.+\mathcal{S}_{4}^{\alpha}(t)\left[A_{n k}^{\dagger} \rho_{k l} A_{l m}^{\dagger}-A_{n k}^{\dagger} A_{k l}^{\dagger} \rho_{l m}\right]\right\}+ \text { H.c. },
\end{aligned}
$$

where

$$
\begin{aligned}
& \mathcal{S}_{1}^{\alpha}(t)=\frac{1}{f \tau_{B}} \int_{t-f \tau_{B}}^{t} d t^{\prime} \bar{s}_{2}^{\alpha}\left(t^{\prime}\right) s_{1}^{\alpha}\left(t^{\prime}\right), \\
& \mathcal{S}_{2}^{\alpha}(t)=\frac{1}{f \tau_{B}} \int_{t-f \tau_{B}}^{t} d t^{\prime} \bar{s}_{1}^{\alpha}\left(t^{\prime}\right) s_{2}^{\alpha}\left(t^{\prime}\right), \\
& \mathcal{S}_{3}^{\alpha}(t)=\frac{1}{f \tau_{B}} \int_{t-f \tau_{B}}^{t} d t^{\prime} \bar{s}_{2}^{\alpha}\left(t^{\prime}\right) s_{2}^{\alpha}\left(t^{\prime}\right), \\
& \mathcal{S}_{4}^{\alpha}(t)=\frac{1}{f \tau_{B}} \int_{t-f \tau_{B}}^{t} d t^{\prime} \bar{s}_{1}^{\alpha}\left(t^{\prime}\right) s_{1}^{\alpha}\left(t^{\prime}\right) .
\end{aligned}
$$

By converting Eq. (B18) into an operator-valued equation we obtain Eq. (19) in the main text.

\section{APPENDIX C: CLOSED SET OF EQUATIONS OF MOTIONS FOR FULL MASTER EQUATION}

A closed set of differential equations for the components of the vector $\boldsymbol{v}=\left(\left\langle X^{2}\right\rangle,\left\langle P^{2}\right\rangle,\langle X P+P X\rangle\right)^{\top}$ with positionmomentum correlator $D=X P+X P$ can be derived from 
the full master equation and is given by

$$
\begin{aligned}
& \frac{d}{d t} v_{1}=\omega_{0} v_{3}+\left\{-i v_{1}\left[h_{1}\left(h_{2}^{*} S_{1}^{\alpha}+h_{2} S_{3}^{\alpha}\right)_{+} h_{1}^{*}\left(h_{2} S_{2}^{\alpha}+h_{2}^{*} S_{4}^{\alpha}\right)\right]+\frac{-i v_{3}+1}{2}\left[h_{2}\left(h_{2}^{*} S_{1}^{\alpha}+h_{2} S_{3}^{\alpha}\right)+h_{2}^{*}\left(h_{2} S_{2}^{\alpha}+h_{2}^{*} S_{4}^{\alpha}\right)\right]+\text { H.c. }\right\}, \\
& \frac{d}{d t} v_{2}=-\frac{\omega^{2}(t)}{\omega_{0}} v_{3}+\left\{i v_{2}\left[h_{2}\left(h_{1}^{*} S_{1}^{\alpha}+h_{1} S_{3}^{\alpha}\right)+h_{2}^{*}\left(h_{1} S_{2}^{\alpha}+h_{1}^{*} S_{4}^{\alpha}\right)\right]+\frac{i v_{3}+1}{2}\left[h_{1}\left(h_{1}^{*} S_{1}^{\alpha}+h_{1} S_{3}^{\alpha}\right)+h_{1}^{*}\left(h_{1} S_{2}^{\alpha}+h_{1}^{*} S_{4}^{\alpha}\right)\right]+\text { H.c. }\right\},
\end{aligned}
$$

and

$$
\begin{aligned}
\frac{d}{d t} v_{3}= & 2 \omega_{0} v_{2}-2 \frac{\omega^{2}(t)}{\omega_{0}} v_{1}+\left\{i v_{1}\left[h_{1}\left(h_{1}^{*} S_{1}^{\alpha}+h_{1} S_{3}^{\alpha}\right)+h_{1}^{*}\left(h_{1} S_{2}^{\alpha}+h_{1}^{*} S_{4}^{\alpha}\right)\right]-i v_{2}\left[h_{2}\left(h_{2}^{*} S_{1}^{\alpha}+h_{2} S_{3}^{\alpha}\right)+h_{2}^{*}\left(h_{2} S_{2}^{\alpha}+h_{2}^{*} S_{4}^{\alpha}\right)\right]\right. \\
& \left.-\frac{1}{2}\left[\left(h_{1}^{*} h_{2}+h_{2}^{*} h_{1}\right)\left(S_{1}^{\alpha}+S_{2}^{\alpha}\right)+2 h_{1} h_{2} S_{3}^{\alpha}+2 h_{1}^{*} h_{2}^{*} S_{4}^{\alpha}\right]-v_{3}\left[S_{1}^{\alpha}-S_{2}^{\alpha}\right]+\text { H.c. }\right\} .
\end{aligned}
$$

In case of the HTME, we obtain

$$
\frac{d \boldsymbol{v}}{d t}=M_{i}^{\alpha} \boldsymbol{v}+A_{w} \cos \left(\omega_{L} t\right) M_{t} \boldsymbol{v}+\boldsymbol{I}_{\alpha}
$$

where

$$
M_{i}^{\alpha}=\left(\begin{array}{ccc}
-2 \gamma \sin ^{2}(\alpha) & 0 & \frac{\gamma}{2} \sin (2 \alpha)+\omega_{0} \\
0 & -2 \gamma \cos ^{2}(\alpha) & \frac{\gamma}{2} \sin (2 \alpha)-\omega_{0} \\
\gamma \sin (2 \alpha)-2 \omega_{0} & \gamma \sin (2 \alpha)+2 \omega_{0} & -\gamma
\end{array}\right)
$$

determines the isolated system dynamics,

$$
M_{t}=\left(\begin{array}{ccc}
0 & 0 & 0 \\
0 & 0 & -\omega_{0} \\
-2 \omega_{0} & 0 & 0
\end{array}\right)
$$

accounts for the external driving, and

$$
\boldsymbol{I}_{\alpha}=\left[2 n\left(\omega_{0}\right)+1\right] \gamma\left(\begin{array}{c}
\sin ^{2}(\alpha) \\
\cos ^{2}(\alpha) \\
-\sin (2 \alpha)
\end{array}\right)
$$

is the inhomogeneity in Eq. (C4).

\section{APPENDIX D: UNITARY TRANSFORMATION LEADING TO $\mathcal{H}^{\alpha}$}

The unitary transformation relating the total Hamiltonian $H^{\alpha}$ to $\mathcal{H}^{\alpha}$ in Eq. (26) is given by

$$
U=e^{-\frac{i}{\hbar} \frac{\sin (\alpha)}{\omega_{0}} x \sum_{r} \kappa_{r} x_{r}} .
$$

This transformation leaves the position operators of the system and bath unchanged, i.e., $x_{r}=U^{\dagger} x_{r} U$ and $x=U^{\dagger} x U$. However, the momentum operators transform as

$$
\begin{aligned}
U^{\dagger} p_{r} U & =p_{r}-\frac{\sin (\alpha)}{\omega_{0}} \kappa_{r} x, \\
U^{\dagger} p U & =p-\frac{\sin (\alpha)}{\omega_{0}} \sum_{r=1}^{N} \kappa_{r} x_{r} .
\end{aligned}
$$

The term $\mathcal{H}_{\text {shift }}$ in Eq. (26) is given by

$$
\mathcal{H}_{\text {shift }}=\left[\frac{1}{2 \omega_{0}^{2}} \sum_{r=1}^{N} \frac{\kappa_{r}^{2}}{m_{r}} x^{2}-\frac{1}{2 m \omega_{0}^{2}}\left(\sum_{r=1}^{N} \kappa_{r} x_{r}\right)^{2}\right] \sin ^{2}(\alpha) .
$$

\section{APPENDIX E: MASTER EQUATION RESULTING FROM $\mathcal{H}^{\alpha}$}

Following the steps in Appendix B, the transformed Hamiltonian $\mathcal{H}^{\alpha}$ gives rise to the following master equation,

$$
\begin{aligned}
\dot{\rho}_{U}= & -\frac{i}{\hbar}\left[H_{\mathrm{S}}^{F}(t), \rho_{U}\right] \\
& +\left\{\mathcal{U}_{1}^{\alpha}(t)\left[A(t) \rho_{U} A^{\dagger}(t)-A^{\dagger}(t) A(t) \rho_{U}\right]\right. \\
& +\mathcal{U}_{2}^{\alpha}(t)\left[A^{\dagger}(t) \rho_{U} A(t)-A(t) A^{\dagger}(t) \rho_{U}\right] \\
& +\mathcal{U}_{3}^{\alpha}(t)\left[A(t) \rho_{U} A(t)-A(t) A(t) \rho_{U}\right] \\
& \left.+\mathcal{U}_{4}^{\alpha}(t)\left[A^{\dagger}(t) \rho_{U} A^{\dagger}(t)-A^{\dagger}(t) A^{\dagger}(t) \rho_{U}\right]+\text { H.c. }\right\},
\end{aligned}
$$

where the operators $A$ are the same as in Appendix B and

$$
\begin{aligned}
& \mathcal{U}_{1}^{\alpha}(t)=\frac{1}{f \tau_{B}} \int_{t-f \tau_{B}}^{t} d t^{\prime} \bar{u}_{2}^{\alpha}\left(t^{\prime}\right) u_{1}\left(t^{\prime}\right), \\
& \mathcal{U}_{2}^{\alpha}(t)=\frac{1}{f \tau_{B}} \int_{t-f \tau_{B}}^{t} d t^{\prime} \bar{u}_{1}^{\alpha}\left(t^{\prime}\right) u_{2}\left(t^{\prime}\right),
\end{aligned}
$$




$$
\begin{aligned}
& \mathcal{U}_{3}^{\alpha}(t)=\frac{1}{f \tau_{B}} \int_{t-f \tau_{B}}^{t} d t^{\prime} \bar{u}_{2}^{\alpha}\left(t^{\prime}\right) u_{2}\left(t^{\prime}\right), \\
& \mathcal{U}_{4}^{\alpha}(t)=\frac{1}{f \tau_{B}} \int_{t-f \tau_{B}}^{t} d t^{\prime} \bar{u}_{1}^{\alpha}\left(t^{\prime}\right) u_{1}\left(t^{\prime}\right) .
\end{aligned}
$$

As in Appendix B, $\tau_{B}$ is the bath correlation time and $f=$ 10. The function $u_{1}$ in Eq. (E2) is given by

$$
u_{1}(t)=-i \sqrt{\frac{\hbar}{2 m \omega_{0}}} h_{2}(t),
$$

where $h_{2}(t)$ is defined in Eq. (A7) and only depends on the parameters of the classical parametric oscillator. Furthermore,

$$
\begin{aligned}
u_{2}(t)=\left[u_{1}(t)\right]^{*} \text { and } \\
\bar{u}_{i}^{\alpha}(t)=\frac{1}{\hbar} \int_{-\infty}^{\infty} d \omega J^{\alpha}(\omega) n(\omega) \int_{0}^{\infty} d \tau e^{i \omega \tau} u_{i}(t-\tau),
\end{aligned}
$$

where $J^{\alpha}$ given in Eq. (29). By calculating the discrete Fourier transform $\mathcal{F}\left[u_{i}\right]$ of $u_{i}$ and neglecting principle value integrals analogous to Eq. (B17), Eq. (E4) can be written as

$$
\bar{u}_{i}^{\alpha}(t) \approx \frac{\pi}{\hbar} \sum_{k=-k_{\max }}^{k_{\max }} J^{\alpha}\left(w_{k}\right) n\left(w_{k}\right) e^{i w_{k} t} \mathcal{F}\left[u_{i}\right]\left(\omega_{k}\right) .
$$

Since $u_{1} \propto h_{2}$ and $u_{2} \propto h_{2}^{*}$, Eq. (E5) shows that the systembath interaction is determined by the spectral density $\boldsymbol{J}^{\alpha}\left(w_{k}\right)$ evaluated at the characteristic frequencies $\omega_{k}=\omega_{c}$ of the classical harmonic oscillator solutions.
[1] H. J. Metcalf and P. van der Straten, Laser Cooling and Trapping (Springer, New York, 1999).

[2] M. Kiffner and M. J. Hartmann, Phys. Rev. A 81, 021806(R) (2010).

[3] M. Kiffner, U. Dorner, and D. Jaksch, Phys. Rev. A 85, 023812 (2012).

[4] S. Diehl, A. Tomadin, A. Micheli, R. Fazio, and P. Zoller, Phys. Rev. Lett. 105, 015702 (2010).

[5] M. Höning, M. Moos, and M. Fleischhauer, Phys. Rev. A 86, 013606 (2012).

[6] S. Diehl, A. Micheli, A. Kantian, B. Kraus, H. P. Büchler, and P. Zoller, Nat. Phys. 4, 878 (2008).

[7] M. Müller, S. Diehl, G. Pupillo, and P. Zoller, Adv. At. Mol. Opt. Phys. 61, 1 (2012).

[8] F. Verstraete, M. M. Wolf, and J. Ignacio Cirac, Nat. Phys. 5, 633 (2009).

[9] C. Capan, K. Behnia, Z. Z. Li, H. Raffy, and C. Marin, Phys. Rev. B 67, 100507 (2003).

[10] R. Mankowsky, B. Liu, S. Rajasekaran, H. Y. Liu, D. Mou, X. J. Zhou, R. Merlin, M. Först, and A. Cavalleri, Phys. Rev. Lett. 118, 116402 (2017).

[11] M. Först, R. I. Tobey, H. Bromberger, S. B. Wilkins, V. Khanna, A. D. Caviglia, Y.-D. Chuang, W. S. Lee, W. F. Schlotter, J. J. Turner, M. P. Minitti, O. Krupin, Z. J. Xu, J. S. Wen, G. D. Gu, S. S. Dhesi, A. Cavalleri, and J. P. Hill, Phys. Rev. Lett. 112, 157002 (2014).

[12] M. Först, A. Frano, S. Kaiser, R. Mankowsky, C. R. Hunt, J. J. Turner, G. L. Dakovski, M. P. Minitti, J. Robinson, T. Loew, M. Le Tacon, B. Keimer, J. P. Hill, A. Cavalleri, and S. S. Dhesi, Phys. Rev. B 90, 184514 (2014).

[13] T. F. Nova, A. Cartella, A. Cantaluppi, M. Först, D. Bossini, R. V. Mikhaylovskiy, A. V. Kimel, R. Merlin, and A. Cavalleri, Nat. Phys. 13, 132 (2017).

[14] A. D. Caviglia, R. Scherwitzl, P. Popovich, W. Hu, H. Bromberger, R. Singla, M. Mitrano, M. C. Hoffmann, S. Kaiser, P. Zubko, S. Gariglio, J.-M. Triscone, M. Först, and A. Cavalleri, Phys. Rev. Lett. 108, 136801 (2012).

[15] M. Först, A. D. Caviglia, R. Scherwitzl, R. Mankowsky, P. Zubko, V. Khanna, H. Bromberger, S. B. Wilkins, Y.-D. Chuang, W. S. Lee, W. F. Schlotter, J. J. Turner, G. Dakovski, M. P. Minitti,
J. Robinson, S. R. Clark, D. Jaksch, J.-M. Triscone, J. P. Hill, S. S. Dhesi, and A. Cavalleri, Nat. Mater. 14, 883 (2015).

[16] M. Först, K. R. Beyerlein, R. Mankowsky, W. Hu, G. Mattoni, S. Catalano, M. Gibert, O. Yefanov, J. N. Clark, A. Frano, J. M. Glownia, M. Chollet, H. Lemke, B. Moser, S. P. Collins, S. S. Dhesi, A. D. Caviglia, J.-M. Triscone, and A. Cavalleri, Phys. Rev. Lett. 118, 027401 (2017).

[17] M. Rini, R. Tobey, N. Dean, J. Itatani, Y. Tomioka, Y. Tokura, R. W. Schoenlein, and A. Cavalleri, Nature (London) 449, 72 (2007).

[18] M. Liu, H. Y. Hwang, H. Tao, A. C. Strikwerda, K. Fan, G. R. Keiser, A. J. Sternbach, K. G. West, S. Kittiwatanakul, J. Lu, S. A. Wolf, F. G. Omenetto, X. Zhang, K. A. Nelson, and R. D. Averitt, Nature (London) 487, 345 (2012).

[19] S. J. Denny, S. R. Clark, Y. Laplace, A. Cavalleri, and D. Jaksch, Phys. Rev. Lett. 114, 137001 (2015).

[20] F. Schlawin, A. S. D. Dietrich, M. Kiffner, A. Cavalleri, and D. Jaksch, Phys. Rev. B 96, 064526 (2017).

[21] D. Fausti, R. I. Tobey, N. Dean, S. Kaiser, A. Dienst, M. C. Hoffmann, S. Pyon, T. Takayama, H. Takagi, and A. Cavalleri, Science 331, 189 (2011).

[22] W. Hu, S. Kaiser, D. Nicoletti, C. R. Hunt, I. Gierz, M. C. Hoffmann, M. Le Tacon, T. Loew, B. Keimer, and A. Cavalleri, Nat. Mater. 13, 705 (2014).

[23] M. Mitrano, A. Cantaluppi, D. Nicoletti, S. Kaiser, A. Perucchi, S. Lupi, P. Di Pietro, D. Pontiroli, M. Riccò, S. R. Clark, D. Jaksch, and A. Cavalleri, Nature (London) 530, 461 (2016).

[24] S. Gröblacher, A. Trubarov, N. Prigge, G. D. Cole, M. Aspelmeyer, and J. Eisert, Nat. Commun. 6, 7606 (2015).

[25] G. A. Álvarez and D. Suter, Phys. Rev. Lett. 107, 230501 (2011).

[26] T. Yuge, S. Sasaki, and Y. Hirayama, Phys. Rev. Lett. 107, 170504 (2011).

[27] S. Gasparinetti, P. Solinas, S. Pugnetti, R. Fazio, and J. P. Pekola, Phys. Rev. Lett. 110, 150403 (2013).

[28] R. Graham and R. Hubner, Ann. Phys. (NY) 234, 300 (1994).

[29] H.-P. Breuer and F. Petruccione, The Theory of Open Quantum Systems (Oxford University Press, Oxford, 2002).

[30] R. Blümel, A. Buchleitner, R. Graham, L. Sirko, U. Smilansky, and H. Walther, Phys. Rev. A 44, 4521 (1991).

[31] S. Kohler, T. Dittrich, and P. Hänggi, Phys. Rev. E 55, 300 (1997).

[32] H.-P. Breuer and F. Petruccione, Phys. Rev. A 55, 3101 (1997). 
[33] B. Donvil, J. Stat. Mech. Theory Exp. (2018) 043104.

[34] M. Floquet, Ann. Sci. l'É.N.S. 12, 47 (1883).

[35] J. H. Shirley, Phys. Rev. 138, B979 (1965).

[36] P. Hänggi, in Quantum Transport and Dissipation, edited by T. Dittrich, P. Hänggi, G.-L. Ingold, B. Kramer, G. Schön, and W. Zwerger (Wiley, New York, 1997), Chap. 5.

[37] C. F. Lo, Phys. Rev. A, At. Mol. Opt. Phys. 43, 404 (1991).

[38] C. F. Lo, Phys. Rev. A 47, 115 (1993).

[39] M. S. Abdalla and R. K. Colegrave, Phys. Rev. A 48, 1526 (1993).

[40] C. M. A. Dantas, I. A. Pedrosa, and B. Baseia, Phys. Rev. A 45, 1320 (1992).

[41] J.-Y. Ji, J. K. Kim, and S. P. Kim, Phys. Rev. A 51, 4268 (1995).

[42] D. Sheng, R. D. Khan, Z. Jialun, and S. Wenda, Int. J. Theor. Phys. 34, 355 (1995).

[43] D.-Y. Song, J. Phys. A. Math. Gen. 32, 3449 (1999).

[44] K. H. Yeon, K. K. Lee, C. I. Um, T. F. George, and L. N. Pandey, Phys. Rev. A 48, 2716 (1993).

[45] C.-I. Um, K.-H. Yeon, and T. F. George, Phys. Rep. 362, 63 (2002).

[46] J.-Y. Ji, J. K. Kim, S. P. Kim, and K.-S. Soh, Phys. Rev. A 52, 3352 (1995).

[47] I. A. Pedrosa, Phys. Rev. A 55, 3219 (1997).

[48] H. Kohler and F. Sols, Phys. A Stat. Mech. Appl. 392, 1989 (2013).

[49] J.-D. Bao and Y.-Z. Zhuo, Phys. Rev. E 71, 010102 (2005).

[50] A. J. Leggett, Phys. Rev. B 30, 1208 (1984).

[51] A. Cuccoli, A. Fubini, V. Tognetti, and R. Vaia, Phys. Rev. E 64, 066124 (2001).

[52] U. Weiss, Quantum Dissipative Systems, 2nd ed. (World Scientific, Singapore, 1999).

[53] C. Gardiner and P. Zoller, Quantum Noise, 2nd ed. (Springer, New York, 2000).

[54] D. W. Hone, R. Ketzmerick, and W. Kohn, Phys. Rev. E 79, 051129 (2009).

[55] M. Bukov, L. D’Alessio, and A. Polkovnikov, Adv. Phys. 64, 139 (2015).

[56] D. Leibfried, R. Blatt, C. Monroe, and D. Wineland, Rev. Mod. Phys. 75, 281 (2003).
[57] M. Abramowitz and I. A. Stegun, Handbook of Mathematical Functions with Formulas, Graphs, and Mathematical Tables (National Bureau of Standards Applied Mathematics Series, U.S. Government Printing Office, Washington, DC, 1972).

[58] E. Wigner, Phys. Rev. 40, 749 (1932).

[59] A. Polkovnikov, Ann. Phys. (NY) 325, 1790 (2010).

[60] C. Zerbe and P. Hänggi, Phys. Rev. E 52, 1533 (1995).

[61] Y. Rezek and R. Kosloff, New J. Phys. 8, 83 (2006).

[62] J. Eisert and M. M. Wolf, in Quantum Information with Continuous Variables of Atoms and Light (Imperial College Press, London, 2007), pp. 23-42.

[63] F. Galve, L. A. Pachón, and D. Zueco, Phys. Rev. Lett. 105, 180501 (2010).

[64] K. W. Chang and C. K. Law, Phys. Rev. A 81, 052105 (2010).

[65] R. Schmidt, A. Negretti, J. Ankerhold, T. Calarco, and J. T. Stockburger, Phys. Rev. Lett. 107, 130404 (2011).

[66] R. Blatt, P. Zoller, G. Holzmüller, and I. Siemers, Zeitschrift für Phys. D Atoms, Mol. Clust. 4, 121 (1986).

[67] C. Zerbe, P. Jung, and P. Hänggi, Phys. Rev. E 49, 3626 (1994).

[68] S. Arnold, J. H. Li, S. Holler, A. Korn, and A. F. Izmailov, J. Appl. Phys. 78, 3566 (1995).

[69] J. T. Stockburger and H. Grabert, Phys. Rev. Lett. 88, 170407 (2002).

[70] R. Schmidt, S. Rohrer, J. Ankerhold, and J. T. Stockburger, Phys. Scr. T151, 014034 (2012).

[71] S. Rahimi-Keshari, T. Kiesel, W. Vogel, S. Grandi, A. Zavatta, and M. Bellini, Phys. Rev. Lett. 110, 160401 (2013).

[72] K. K. Sabapathy, Phys. Rev. A 93, 042103 (2016).

[73] H.-B. Chen, C. Gneiting, P.-Y. Lo, Y.-N. Chen, and F. Nori, Phys. Rev. Lett. 120, 030403 (2018).

[74] Arvind, N. Mukunda, and R. Simon, Phys. Rev. A 56, 5042 (1997).

[75] H. R. Lewis and W. B. Riesenfeld, J. Math. Phys. 10, 1458 (1969).

[76] K. F. Riley, M. P. Hobson, and S. J. Bence, Mathematical Methods for Physics and Engineering, 3rd ed. (Cambridge University Press, Cambridge, 2006), p. 1359. 